\title{
Design Leadership: Three Pedagogical Pairings for Performative Practice
}

Who will lead in the much-needed awakening to the problem of the divide-and-conquer mentality that has strapped the construction industry to vast inefficiencies BOTH in the way buildings are conceived, designed, delivered AND how they perform? And in the barrage of contemporary claims for performance-based design - what is the fate of "Design"?

This paper examines these questions with examples of substantial and emerging pedagogical initiatives that are critically founded and practically executed with a view toward a more integrated and better-designed future - of performative built environments and the practices that produce them.

\section{PRACTICE}

The world is an increasingly complicated and ever changing place. In guarding its turf architects, the buildings they produce and architectural education have not always kept pace, and when the profession fails to keep pace, entrepreneurs whether they be developers, construction managers, design builders, or renderers on distant shores stand ready to fill the gap. With the increasingly diminished, parceled role of the architect, from the once conceiver, developer and party in charge of the project delivery (construction) through whom all decisions passed - to conceptual designer (form maker) who lacks significant understanding or control over the development and delivery of progressively more complex building systems, projects are increasingly a testament to the quality (or lack thereof) of construction management coordination between discreetly layered trades and disciplines, not the comprehensive design of the architect. Integrated solutions: the ceiling as plenum and light fixture, curtain wall as shading device and radiator, structure as skin and thermal mass, etc., IF conceived at all, are difficult to realize in an industry structured around siloed disciplines, manufacturers and suppliers of off the rack guaranteed components ready to be specified and installed. Design intelligence, that uncanny ability to see through the clutter, integrate, innovate and even invent holistic, seemingly (though undoubtedly not) simple solutions gives way to the coordination of dissimilar standalone parts executed by a construction manager whose risk aversion (playing defense) is "key" to a long success. 
In spite of the conservatism of mainstream architectural practice an emerging (but not so novel) approach to building design spawned by ambition to design "the new" and "the performative" has promulgated partnerships between architects and engineers, industry and educators, even scientists and constructors in the design and production of next-generation built environments (emphasis on environments). They (Foster, FOA, Ito, SHoP, SOM, Buro Happold, ARUP, Schlaich Bergermann and Partners, Atelier 10, Transolar, etc.) focus on operative, not static constructs that integrate systems and performances with material, form and structure to produce places for people. Their approach to projects is multi-scalar, crossdisciplinary and relies on collaboration. Accepting new mandates, tools, and possibilities progressive practices seek to design and realize ever more performative and compelling built environments. They are less concerned with stylistic canon operate out of a desire to design and deliver next generation buildings - realizing that built environments are complicit in society, human and institutional enterprise and will be around for a considerable time.

The quest to realize progressive designs in the context of heightened expectations, expanding regulations, and new technologies on the one hand, and the constraint of materials, available technologies, skills and budgets on the other fuels the need to consider aspects of buildings that conventional or form-making practices might consider either 'too risky' or simply 'uninteresting'. In practice, the need to incorporate 'yet one more thing', by concealing, celebrating, coordinating or integrating it to fulfill (or enhance) the design intent inevitably leads to an enlarged awareness, expanded vocabulary and greater respect for associated disciplines and their agendas. Creative solutions and new possibilities are ultimately borne out ambition that is bounded by restraint.
As for myself, I experience a sort of terror when, at the moment of setting to work and finding myself before the infinitude of possibilities that present themselves, I have the feeling that everything is permissible to me ... Will I then have to lose myself in this abyss of freedom? ... Let me have something finite, definite - matter that can lend itself to my operation only insofar as it is commensurate with my possibilities. As such matter presents itself to me together with its limitations, I must in turn impose mine upon it. ${ }^{1}$

- Igor Stravinsky

\section{EDUCATION}

But this is not substantially (or easily) taught in most architectural educational programs which guard the profession by emphasizing knowing what we already know and how to do it [design] in isolation over, 1) how to think critically, 2 ) how to find out, and 3) how to collaborate. Just as best practices ${ }^{2}$ wrestle to design increasingly complex performative environments with expanded interdisciplinary design teams engaging associative tools, techniques and novel ways of thinking - interdisciplinary associations, tools, and techniques are also needed in the education of the architect (and engineer). A growing number of forward-looking practices and educational initiatives seek to build a horizontal awareness of and respect for other disciplines as collaborators [co-laborers], rather than as merely assistant technologists.

An ever-increasing number of architecture schools (Rensselaer, Yale, Architectural Association (AA), University of Stuttgart, University at Bath, University of Pennsylvania, Syracuse University) recognize this and have structured interdisciplinary experiences into the curriculum and employ working professionals to teach. Many schools include engineers on architecture studio design reviews but few have truly opened the possibility of conspiring together on design and research initiatives designed to develop aptitudes that facilitate effective col- 
laboration and result in the intelligent design and integration of systems - whether structural skins, passive environmental design strategies or as social or cultural protagonists. Business as usual is not an option. Practices that desire to operate according to these new mandates and schools that value them must reform conventional ways of working and teaching in disciplinary isolation. Curricular transformation is critical to reforming the profession and ultimately, its relevance and value to society.

\section{CREATING A DIALOGUE}

In the tide of rising complexity, strategic educational initiatives that create dialogue through interdisciplinary seminars, studios, and workshops led by engineering or architecture professors of practice are needed. Travelling workshops focusing on progressive practices and exemplary work that rely upon interdisciplinary collaboration provide awareness through first hand exposure. Design research initiatives linking practicing architects and engineers with students, consultants, and scientists in the collaborative design of next-generation building integrated systems add educational value and produce content that would not easily occur within the restrictions of practice. In each case engaging diverse experts, each with an awareness of the critical value of design AND other intelligences in imagining, developing and realizing innovative and compelling performative architectures is critical to preparing students for next-generation leadership in the profession.

\section{THE ROLE OF EXPERTISE}

The marriage of disciplinary know-how and design expertise is key to progressive design practice. The scientific method of isolating and solving the problem, has transformed the world in recent centuries, for better AND worse. In the past 150 years the scientific and industrial revolutions have resulted in uncountable and extraordinary advances and new systems of production. They have influenced how we organize and execute AND have even influ- enced how we think. They have birthed new disciplines with greater focus and specificity, especially in the academy where research culture has resulted in disciplinary and departmental structures that reward highly refined expertise in specific well-defined fields of inquiry. In contrast, architectural education has embraced a project/studio-based model dedicated to the development of lateral thinking and synthetic capacity. But in the increasingly complex world of performance expectations how should design expertise be taught, developed and deployed?

In manufacturing, where things are designed, made and distributed, pragmatics and the need to coordinate 'complicate' the problem. As a result industry is adept at communicating and managing diverse sets of knowledge to realize its objectives. But the architecture discipline and construction industry are not the same. In addition to designing "one-off" structures unique to their program and site, buildings have become profoundly complicated - with transport systems, sensors and alarms, fireproofing, lighting and ventilating, sprinklers and smoke exhaust systems, air conditioning, acoustic and environmental considerations, codes, and sustainability standards etc., yet for the most part these are coordinated, not integrated. Alongside the development of myriad systems designed to perform particular tasks have emerged ways of working and thinking about buildings in discreet layers appropriated and reserved for unique tasks - the domain of unique disciplines: the structural zone, the façade zone, the fireproofing zone, the HVAC zone, the sprinkler zone, the lighting zone, etc. Each unique system is designated to a correspondingly unique zone - eliminating any conflict, while also reducing the need to communicate or collaborate in the design and construction phases. By rendering the operations discreet - the expertise, responsibility, physical space, installation and/or maintenance of one system never impact the other, and liability is clear. Where systems must interface is precisely where the processes and performances are 
most likely to break down - in large part because there was no culture of communication or common language.

Managing the vastly increased complexity of the design, installation and/or maintenance of building systems (which is outside the capacity of any one person) has largely become the sum of uniquely isolated problems (i.e. cooling) emanating from uniquely isolated disciplines (HVAC engineers) without a structured mechanism for understanding the impact of one system on the others. Critical Path Method (CPM) of scheduling provides a vivid illustration. The grouping and sequencing of tasks, and which are critical in advance of others, leads to a series of subprojects (critical paths) free of interference from another. The construction manager's role, to coordinate the expertise (designs, systems and tasks) - is too often executed under the moniker of integration.

\section{DESIGN 'IS' AN EXPERTISE}

Integration has come to mean the successful interplay of multiple components or disciplines - where two or more things work together successfully (don't interfere with each other) however, there is another more fundamental definition - how one component can perform multiple tasks. The case for integrated (designed) solutions over coordinated ones, operational buildings over static ones, built ecologies over built anomalies, smart buildings over strong ones relies on an economy of means and intelligence that must cross disciplinary boundaries, trade boundaries and intellectual boundaries. Integration depends on design expertise and leadership capable of managing and optimizing complex multivariable problems creatively and constructively.

Progressive design results from an ecology of the mind (Bateson) - an awareness and openness to the mutual influences of multiple variables that may reside in the background or rise to the fore, but which are nevertheless mutually influential - much like a building and its occupants. It re- sults from an attitude that listens and constructs - neither completely willfully or slavishly, but with the authority of a generous, inclusive and open mind. It comes from design leaders who have a vision that transcends the merely visual or formal, understands the importance of that vision, knowing that it is robust enough and the collaboration team clever enough to absorb and effectively integrate all the criteria it must creatively manage. Understanding and appreciating the need to creatively integrate the many complexities of a building project cannot be assumed - it must be learned.

Exposure and experience are invaluable. Realizing projects through the development and construction phases is an irreplaceable schoolmaster that broadens the perspective and awareness of myriad little, but critical aspects of designing, constructing and operating a building. Early projects often result in disappointments - those things not drawn or highly regarded: material properties, wind, noise, vibration, product availability, smoke alarms, exit signs, building signage and the paths to them, etc. but if we do not learn to respect them, consider them, understand them - they will undoubtedly intrude. The question for educators is how to nurture awareness, aptitudes and skills that prepare students to lead effectively in this new world of complexity and collaboration when they are not yet in it?

Three proposed pedagogical pairings challenge traditional scholastic settings and instructor qualifications in favor of adding (not replacing the classroom/studio and professorate) broader contexts, exposures and experiences to raise awareness of pragmatic resistances that fuel Stravinsky's creativity. Just as every syllabus, curriculum or teacher is not a good one, any experience meeting the basics of these pairings will also not do - we have to remember the reason, and engage only those contexts and persons who strive to integrate, who understand ecological thinking and approaches and believe in progressive possibilities with positive human and environmental consequences. 
THREE PEDAGOGICAL PAIRINGS

Three Pedagogical Pairings that take students out of conventional settings are called for in the education of the architect for $21^{\text {st }}$ century progressive practice. Pairings between:

1. Architecture and Other Disciplines (engineering, science, and/or humanities: linking design culture to other sets of expertise and parameters)

2. Design and Research Cultures (linking speculative approaches and research methodologies), and

3. Education and Practice (leveraging the complementary virtues of thought and action; those with the opportunity to speculate and research, and those with the mandate of manifesting complex built projects)

\section{Architecture and Other Disciplines}

Schools should structure required, synthetic interdisciplinary studios, seminars and/or out-of-classroom experiences staffed by instructors from diverse disciplines.

Performance-based design of built environments best occurs in fertile collaboration with other disciplines - integrating expertise beyond that of the architect who must learn to master his/her own unique coordinating and leadership role [expertise]. Daring to engage unfamiliar interdisciplinary associations opens manifold possibilities: of achieving the economy and elegance of true integration over coordination, learning from one another and conceiving of potential opportunities that could not have been predicted in isolation. But to effectively engage interdisciplinary collaborations a designer must have a reasonable understanding of first principles - sufficient enough to understand (conceptually - not numerically) their collaborator's work. Understanding building science, and more importantly the first principles underlying it, are essential. This too must reside in the curriculum and be valued by all, not as a mere matter of knowledge or skill e.g. what is a moment force, or how to do a heat-loss calculation - but as a matter of understanding the principles at work. $\mathrm{S} /$ he must also have experience with and respect for engineers and scientists who are also curious about the world and searching for applications and answers. And ultimately, there must be a project that calls for multiple expertises. This can be challenging to construct in the academy.

It is only logical, though certainly not trivial; to engage closely allied engineering disciplines in the design education enterprise. Though virtually every building project requires an interface with structural and mechanical engineers, mainstream practices rarely engage them in a truly interdisciplinary mode and educational institutions rarely engage them at all. There are exceptions and forward looking architecture programs find substantive ways to integrate engineering faculty, students and professionals in the educational experience through:

- Interdisciplinary comprehensive design studios

- Interdisciplinary workshops focused on specific problems

- Focused interdisciplinary research studios

- Interdisciplinary research settings

In each of these scenarios the integration of engineering faculty and/or students breaks the isolated architecture studio bubble to expose students ( $A$ and $E$ ) to greater possibilities, alternative agendas and languages. When well structured and lead, interdisciplinary initiatives stimulate a constructive conversation capable of inspiring mutual respect and confidence, sharpening communication and ideas, and enhancing creativity and imagination.

Specialty consultants - lighting, acoustics, façade specialists etc., are often treated as those who retrofit 'their system' to a 
preconceived architectural design, but they could influence it. Integrating expertise at the early stages of a design does not merely benefit the results, but also - and more importantly in an educational setting - it develops a student's broader understanding and respect for other disciplines and how collaborators can assist both in concept resolution AND its formation. Focused interdisciplinary studios dedicated to room acoustics [soundscapes], the radical transformation of space and perception by light, or façade performance in relation to programmatic, environmental and occupation criteria (to name just a few examples) provide fertile learning settings for students, faculty and the professionals involved.

Initiatives pairing multiple disciplines are not new to academia. Mohsen Mostafavi, in his initiative to shift the Architectural Association from a focus on the avant-garde to a kind of progressive mainstream project, brought practicing architects and engineers into the den - to further the conversation and stimulate innovation. The University of Pennsylvania engaged former ARUP Advanced Geometries Unit Director Cecil Balmond to broaden and stimulate the discourse. Under Mark Robbins, Syracuse University instituted the Transdisciplinary Media Studio [TdMS] with the mission of transforming thinking about the future of collaboration. The initiative engages one architect and two interdisciplinary collaborators in studio settings focused on design research projects. Rensselaer Polytechnic Institute engages practicing engineers, engineering faculty and students in a required comprehensive design studio. The Bedford Initiatives [Rensselaer Polytechnic Institute] feature interdisciplinary $A / E$ seminars and an annual $A / E$ travelling workshop that examines exemplary architecture and engineering practices and their projects' worldwide.

On more speculative and forward-looking design undertakings even more diverse expertise may be required - from the physicist to the hydrologist, biologist, chemist, botanist, econo- mist, or geologist, etc. While broadly integrated initiatives may not occur as often on contracted building design projects, or design studios and seminars, they remain critical to the second important pairing - design and research.

\section{Design and Research}

Schools should integrate a required design-research experience into the curriculum

There has been much discussion about what is meant by design research. I will use a relatively simple framework that qualifies something as research when, it is an undertaking by which we aim to increase our knowledge. ${ }^{2}$ It is a search for something, not how to validate or mimic it. Validation and mimicry have their place, but with respect to education, addressing open questions is critical. Looking beyond the metaphor or anything with apriori solutions, an inquiry qualifies as research when it can fail and is not merely figuring something out, or solving a problem.

The reasons for pairing research and design in architectural education are manifold: to produce lifelong learners, to provoke the curiosity of investigators in search of integrative possibilities, and to expose students to alternative ways of thinking and working. Research culture, the kind familiar to scientists and engineers, is one that provides a rigor of thinking and action that sharpens language and precision of thought. Understanding research culture in its various kinds, from basic and fundamental to applied and speculative is invaluable to students seeking to develop creative capacity and launch a progressive career. First, students will learn that they must (and have full permission to) act within the laws of nature. It reveals the rigor and specificity of simulation, the experiment, the prototype, and proof of concept peer review, viability, analysis, application and speculation - all critical to exercising the design mind in ways that open the imagination while guarding against sloppy assumptions.

Design expertise and research expertise can be mutually beneficial. It should not be surprising that science and engineering cultures 
provide many examples of research in the academy, but it should also not be surprising that the lateral thinking of architects and schools of architecture have often forged, though not always capitalized on, robust and productive research initiatives.

The Institute of Lightweight Structures [ILEK] at the University of Stuttgart was founded by Architect Frei Otto and continues under the successive leadership of German Architect and Engineer Werner Sobek. Its interdisciplinary enterprise is dedicated to "uniting the aspect of design that is dominant in architecture with the focus on analysis and construction from structural engineering as well as materials science." Though it has contributed much to knowledge about structures and materials, the Institute is not directly connected to the engineering or architecture educational programs at and impacts only a small percentage of its students. The Media Lab is a 1980 product of MIT's Architecture Machine Group. Co-founder Nicholas Negroponte understood the extraordinary potential of interdisciplinary engagement and the digital revolution. While its linkage to the architecture discipline is not direct, it is founded and thrives on the creative spirit and potential of interdisciplinary research settings. Each emerged from an enlightened design intelligence interested in particular questions and challenges and possibilities beyond what was known and in each case the initiatives provoked substantial forward looking research.

The Center for Architectural Structures and Technology (CAST), at the University of Manitoba, founded and directed by Mark West is both a research lab and a resource of the School of Architecture dedicated to "the exploration of architectural possibilities through making, experimentation, invention and discovery." In similar fashion an increasing number of architecture schools have created digital fabrication labs focused on geometry, materiality and fabrication techniques, but one of the most cross-cutting examples of design-led research initiatives is Rensselaer's Center for
Architecture Science and Ecology (CASE) Directed by Professor Anna Dyson.

In collaboration with SOM, CASE brings all three pedagogical pairings together in one interdisciplinary research center focused on the development of next generation building systems. The Center is linked to the practice of SOM through shared research projects and personnel and often engages SOM projects as test-beds for research. Building projects also provide opportunities for the deployment and testing of design research prototypes. CASE's Built Ecologies program teaches both graduates (Masters and PhD) and undergraduate students in interdisciplinary research-based studios. It structures links between undergraduate architecture students and $\mathrm{PhD}$ research projects. Interdisciplinarity is fundamental to its mandate resulting from unique sets of varied expertise linked to each research project, and diverse graduate students who arrive with a variety of educational backgrounds ranging from architecture to engineering and the sciences. Design expertise is paramount, not as the authority as much as it is employed to create vision and manage processes with an eye and discipline for integration and design. What it looks like, how well it is integrated, and how it performs each matter.

\section{Education and Practice}

Schools should structure opportunities to interface with practice and build into the curriculum

The third pairing critical to a progressive ecology of the mind and robust architectural education for the $21^{\text {st }}$ century involves learning in action. Experiential learning best happens in the trenches, on the site, and in the negotiation, not in the classroom. For that reason, the education of an architect is comprised of multiple components education, internship, and examination. But is the emphasis correct? Are 120 hours (out of 5600) of required IDP internship time in 'construction phase observation' sufficient, and is there a critical context in which to discuss it? Is the option (not the requirement) 
to work in construction sufficient? And is the fact that education and internship take place independently and/or in series, (not integrally) the most effective? I argue that they are not. When linked with a good teacher education has great potential to inform 'the trenches', and conversely, 'the trenches' have great potential to inform and enliven both the studio/ classroom and the research/lab.

There is much good to be said for the academically integrated design-build project or internship - as long as there is a clear and progressive educational research objective. Several excellent examples include:

- Rural Studio at Auburn University with the mandate of serving the people and place of Hale County. The design-build initiative engages undergraduates in the design and construction of structures with highly restricted means.

- The Design Build Initiative at Carnegie Mellon directed John Folen has students take on, design and deliver built projects on time and in budget for the community. Failure is an option.

- The pavilion project at the AA provides students an opportunity to speculate, design and realize a project at full scale with engineering and technical assistance from ARUP engineers.

- Valparaiso's Open City, provides an experimental living, working and learning space "to test the relation between an idea and its realization." In operation since the 1950's, it remains a testament to the possibility of integrating full scale making into the curriculum.

\section{- The Solar Decathlon provides a} meaningful opportunity for schools to engage in a full-scale project that is by necessity also interdisciplinary and research and design based.
The multi- semester and multi-year effort required of many of design-build projects can make participation and student learning outcomes difficult to integrate and manage consistently, but there is no real substitute. Integrated Co-op programs and structured internships can also be effective. At Waterloo University (and many) Canadian Schools of Architecture) alternate study and work terms introduce students to practice while inherently creating a temporal relationship between the two contexts. The University of Cincinnati's internship program adds the formal requirement of student reflection on the internship activity. Integrated internships are invaluable when structured to join thinking and speculation with the restraints inherent to project realization.

\section{CONCLUSION}

The Beaux Arts model, the Polytechnic model, the Bauhaus Model, and the Atelier Model are each unique products of their time: Beaux Arts reaffirming culture and beauty - operating according to the canons of proportion and style, the Polytechnic linking science culture with shop culture to extend and refine possibility for the purpose of creating a new world, the Bauhaus integrating craft and design at all scales in search for a total work of art - favoring design over style, making all things functional, elegant and lacking superfluous ornament, and the Atelier model, enmeshing its students in practice under a master architect. But none alone address the need to educate designers to engage and lead the multidisciplinary teams required of next generation built environments. Buildings are evolving - from static structures to complex operative systems and need creative leadership more than ever.

Design leadership IS an expertise. As the conductor gives the symphony structure, tempo, color and voice - hears each instrument, and the whole, architectural design expertise and leadership are needed. The architect (like the conductor) does not play any single instrument as expertly as those in the symphony, but his/ 
her role as an integrator and coordinator makes the whole come together. It is a performance of many that relies on each one.

There is a bright future for the architecture profession but it does not reside in hermetic isolation. The risk of subjugating its larger leadership role and potential to a narrow focus on shape giving and style (though not unimportant) is very real. Many outside the profession stand ready to fill the gap and assume the authorship of the performance, content and coordination of the whole. As the world changes, the Architecture profession should not only keep pace but also lead. In preparation, the studio, the lab, and full-scale projects/sites can each play a significant didactic role that cannot be filled by the other. We should ask, why do Renzo Piano, Rafael Viñoly and Von Gerkan Marg and Partners have their own in-office "schools"? - to indoctrinate - perhaps, but also to inform students of architecture how the integrated model of design works. The challenge for Schools of Architecture that are serious about graduating leaders prepared to anticipate, adapt and operate as change agents in future practice is to create and integrate initiatives that are interdisciplinary, engage research and involve full-scale making and interface with practice.

\section{BIBLIOGRAPHY}

Aayer, Netanel, "The Open City Valparaiso Chile" Architecture of Israel Quarterly Valparaiso, No.53, May, 2003.

Allen, Stan (2000). Practice: Architecture, Technique and Representation (Critical Voices in Art, Theory \& Culture). Routledge, 2000.

Glanville, R (1980) “Why Design Research". Jacques, R and Powell, J (eds) 'Design: Science: Method', Westbury House, Guildford

Bateson, Gregory (1972). Steps to an Ecology of Mind: Collected Essays in Anthropology, Psychiatry, Evolution, and Epistemology. University Of Chicago Press.
Stravinsky, Igor (1942) Poetics of Music: In the Form of Six Lessons. Harvard University Press.

CAST:: The Centre for Architecture Structures \& Technology. web n.d.

Institut fur Leichtbau Entwerfen und Konstruiren, (ILEK) web n.d.

Rural Studio:: Auburn University, web n.d.

\section{ENDNOTES}

1. Igor Stravinsky, Poetics of Music: In the Form of Six Lessons. Harvard University Press, 1942.

2. "Best practices" refers to progressive firms interested in the interiority, exteriority and performance of buildings relative to environmental, institutional and human concerns, their specific context and program - practices that seek innovative integrated design solutions.

3. Ranulph Glanville: Re-Searching Design and Designing Research" self published and revised from Glanville, R (1980) "Why Design Research". Jacques, R and Powell, J (eds) 'Design: Science: Method', Westbury House, Guildford. 